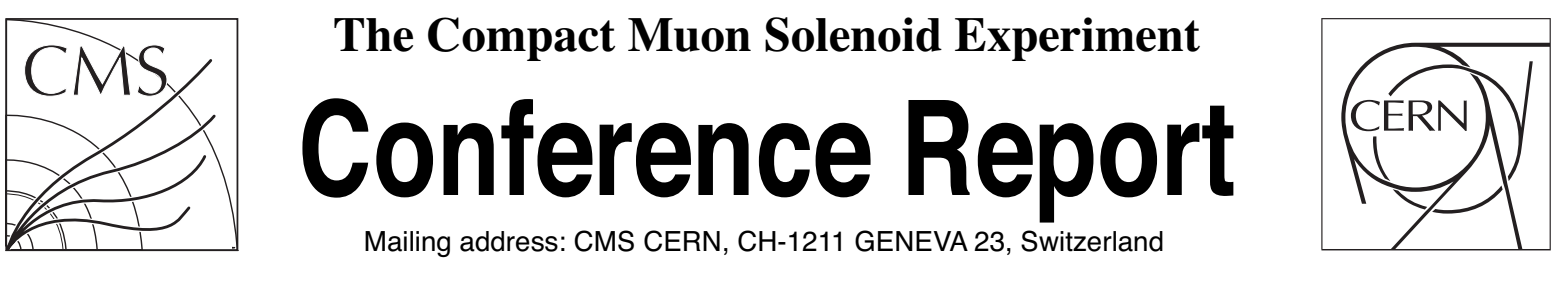

14 December 2018 (v3, 29 January 2019)

\title{
The CMS Tracker Upgrade for the High Luminosity LHC
}

Giacomo Sguazzoni on behalf of the CMS tracker group

\begin{abstract}
The LHC machine is planning an upgrade program which will smoothly bring the luminosity to about $5-7.5 \times 10^{34} \mathrm{~cm}^{-2} \mathrm{~s}^{-1}$ in 2028 , to possibly reach an integrated luminosity of $3000-4500 \mathrm{fb}^{-1}$ by the end of 2039. This High Luminosity LHC scenario, HL-LHC, will require an upgrade program of the LHC detectors known as Phase-2 upgrade. The current CMS Outer Tracker, already running beyond design specifications, and CMS Phase-1 Pixel Detector will not be able to survive HL-LHC radiation conditions and CMS will need completely new devices, in order to fully exploit the highly demanding conditions and the delivered luminosity. The new Outer Tracker should have also trigger capabilities. To achieve such goals, R\&D activities have explored options for both the Outer Tracker and for the Inner Tracker. The solutions developed will allow to include tracking information in the first level trigger stage. The design choices for the Tracker upgrades are discussed along with some highlights on technological approaches and R\&D activities.
\end{abstract}




\title{
The CMS Tracker Upgrade for the High Luminosity LHC
}

\author{
Giacomo Sguazzoni on behalf of the CMS tracker group
}

\begin{abstract}
The LHC machine is planning an upgrade program which will smoothly bring the luminosity to about $5-7.5 \times$ $10^{34} \mathrm{~cm}^{-2} \mathrm{~s}^{-1}$ in $\mathbf{2 0 2 8}$, to possibly reach an integrated luminosity of $3000-4500 \mathrm{fb}^{-1}$ by the end of 2039. This High Luminosity LHC scenario, HL-LHC, will require an upgrade program of the LHC detectors known as Phase-2 upgrade. The current CMS Outer Tracker [1], already running beyond design specifications, and CMS Phase-1 Pixel Detector [2] will not be able to survive HL-LHC radiation conditions and CMS will need completely new devices, in order to fully exploit the highly demanding conditions and the delivered luminosity. The new Outer Tracker should have also trigger capabilities. To achieve such goals, $R \& D$ activities have explored options for both the Outer Tracker and for the Inner Tracker. The solutions developed will allow to include tracking information in the first level trigger stage. The design choices for the Tracker upgrades are discussed along with some highlights on technological approaches and $R \& D$ activities.
\end{abstract}

\section{INTRODUCTION}

The High Luminosity phase of the LHC (HL-LHC) is expected to start in 2026. With an instantaneous luminosity of $5-7.5 \times 10^{-34} \mathrm{~cm}^{-2} \mathrm{~s}^{-1}$ already available in 2028 , the aim is to collect between $3 \mathrm{ab}^{-1}$ and $4.5 \mathrm{ab}^{-1}$ of integrated luminosity per experiment in about ten years. The HL-LHC will operate at a centre-of-mass energy of $14 \mathrm{TeV}$ with a bunch crossing spacing of $25 \mathrm{~ns}$. The number of pile-up (PU) collisions will reach an average of $\sim 140$, increasing to $\sim 200$ in the best performance scenario. The radiation levels will be unprecedented, especially in the innermost layers of the detector, where a fluence of $2.3 \times 10^{16}\left(1 \mathrm{MeV} n_{\mathrm{eq}}\right) / \mathrm{cm}^{2}$ and a total ionizing dose of $12 \mathrm{MGy}(1.2 \mathrm{Grad})$ are foreseen for the baseline integrated luminosity of $3 \mathrm{ab}^{-1}$. The present Outer Tracker [1] and Phase-1 Pixel detector [2] would be unable to cope with the HL-LHC environment [3] due to the significantly higher rate, the longer latency of the upgraded trigger system, that is crucial for operation at high luminosity, and the fatal consequences of the irradiation. The entire Tracker will thus be replaced [4] during the long shutdown in 2024-2025 (LS3) when also LHC will be upgraded. The new Tracker, similar to the current one, will be made up of two subdetectors: the Outer Tracker (OT) and the Inner Tracker (IT).

Despite the foreseen improvements, the muon and calorimeter-based lowest level trigger (Level-1 or L1) will not be able to cope with the contamination due to ghosts generated by the PU and the limited resolution: e.g., in the muon case, no transverse momentum $\left(p_{\mathrm{t}}\right)$ threshold is sufficiently effective

Manuscript received December 20, 2018.

G. Sguazzoni is with Istituto Nazionale di Fisica Nucleare - Sezione di Firenze, I-50019 Sesto Fiorentino (FI), Italy; contact info: https://phonebook.cern.ch/phonebook/\#personDetails/?id=431400 in reducing the rate. The Tracker is then required to provide high-resolution, high-purity, high- $p_{\mathrm{t}}$ tracks to the L1 system.

The main requirements for the Tracker upgrade [3] can be summarized as follows:

- radiation tolerance and cold $\left(-20^{\circ} \mathrm{C}\right)$ operation to be functional up to $3 \mathrm{ab}^{-1}$ (with margins to comply with the best performance scenario);

- increased granularity (occupancy $<\mathcal{O}(1 \%)$ for the OT and $<O(0.1 \%)$ for the IT) and optimized layout for robust pattern recognition;

- reduced passive material;

- track trigger capabilities to contribute to L1;

- large readout bandwidth and deep front-end buffers for higher rate $(750 \mathrm{kHz})$ and the long latency $(12.5 \mu \mathrm{s})$ of the upgraded L1 trigger system;

- extended coverage up to $|\eta| \sim 4$ for efficient PU mitigation and better physics objects reconstruction in the forward region;

- very forward part of IT usable as a luminosity monitor;

- IT fully accessible for maintenance and part replacement.

The layout of the upgraded Tracker is shown in Fig. 1.

\section{THE INNER TRACKER}

The Inner Tracker consists of $\sim 4.9 \mathrm{~m}^{2}$ of silicon pixel detectors for a total of $2 \mathrm{G}$ readout channels. The IT is build up of two types of hybrid pixel modules with $1 \times 2$ or $2 \times 2$ readout chips (ROC), organized into four barrel layers (TBPX) plus eight small disks (TFPX) and four large disks (TEPX) per side (see Fig. 2). A quarter of the mechanical implementation of the IT is shown in Fig. 3.

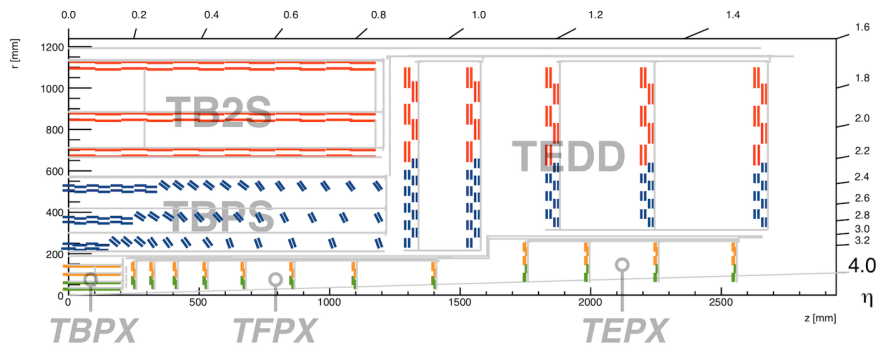

Fig. 1. The layout of the upgraded tracker with labels indicating the various subsystems. Red and blue lines represent OT $2 \mathrm{~S}$ and PS modules respectively. Yellow and green lines represent IT $2 \times 2$ and $1 \times 2$ modules respectively. 

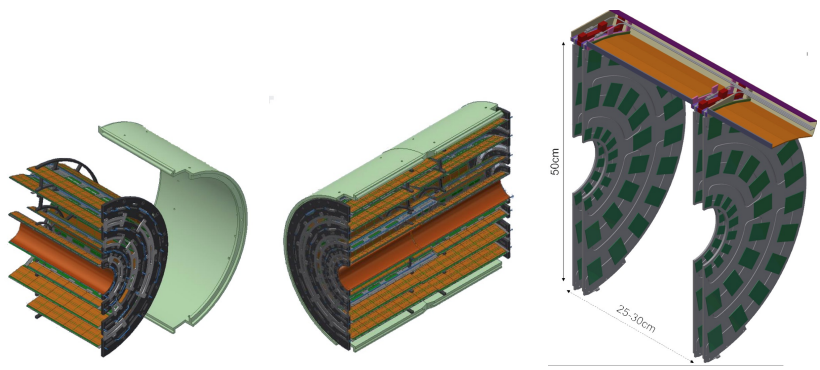

Fig. 2. CAD drawings of the IT parts: half TBPX barrel (left); full TBPX barrel (center); two forward half disks on the support structure (right).

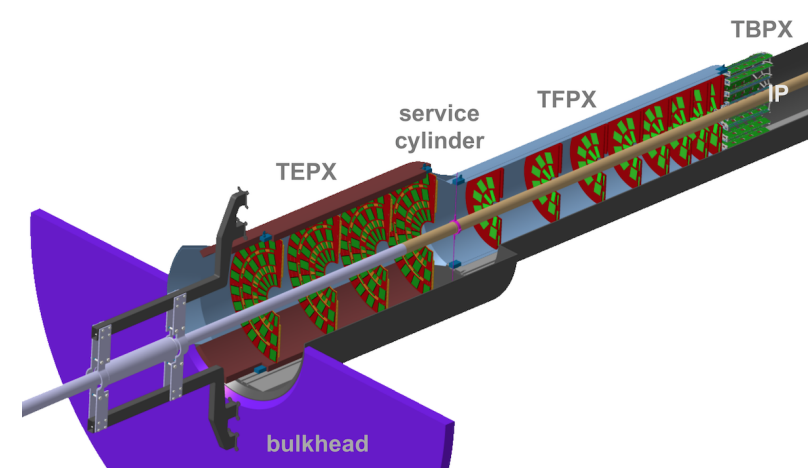

Fig. 3. A sketch of the IT mechanical structure showing the various subparts.

\section{A. IT Pixel Sensors and Readout Chip}

Thin planar n-in-p sensors, with $100-150 \mu \mathrm{m}$ maximum active thickness, have been chosen for the IT to have more stable charge collection efficiency after irradiation [5]. Nevertheless they need rather high bias voltage (up to $0.8-1 \mathrm{kV}$ ) and spark protection between the ROC and the sensor. The alternative option of 3D sensors is still under consideration for the innermost layer since this kind of sensor is potentially more rad-hard, but requires a more complex fabrication and has larger cell capacitance [5]. A sketch illustrating the different concept of the two options can be seen in Fig. 4.

Two possible pixel aspect ratios are being scrutinized for the IT: $25 \times 100 \mu \mathrm{m}^{2}$ (current baseline) and $50 \times 50 \mu \mathrm{m}^{2}$. The bond pad pattern stays the same as shown in Fig. 5 .
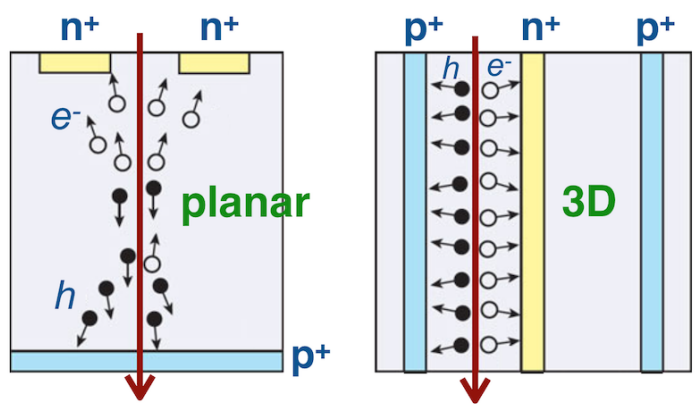

Fig. 4. Illustration of the planar and 3D pixel sensor concepts.
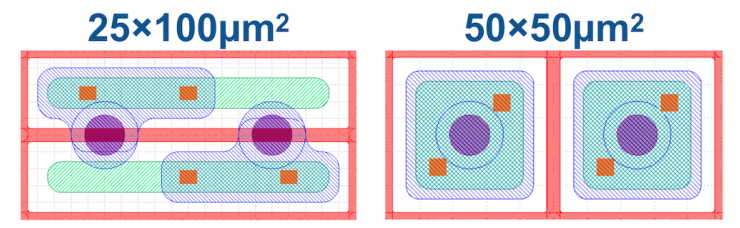

Fig. 5. The $25 \times 100 \mu \mathrm{m}^{2}$ and $50 \times 50 \mu \mathrm{m}^{2}$ pixel cells.

The ROC needed for the IT is a technology frontier device that needs to withstand $1.2 \mathrm{Grad}$ of total ionising dose and $3.2 \mathrm{GHz} / \mathrm{cm}^{2}$ of maximum hit rate. It is being developed by the RD53 collaboration [6] in 65nm technology and features a $50 \times 50 \mu \mathrm{m}^{2}$ elementary cell, low threshold $\left(<1000 e^{-}\right)$, high hit and trigger rate (thanks to up to four $1.28 \mathrm{~Gb} / \mathrm{s}$ output links), radiation resistance and serial powering capabilities [7].

RD53A is the first prototype (about half in size with respect to the foreseen final chip) and it is also being used for the IT R\&D. The final chip submission is foreseen in June 2019. Figure 6 shows a schematic layout of the ShuntLDO, the heart of the serial powering capability of the ROC [7]. A simplified floorplan of the RD53A prototype is shown in Figure 7.

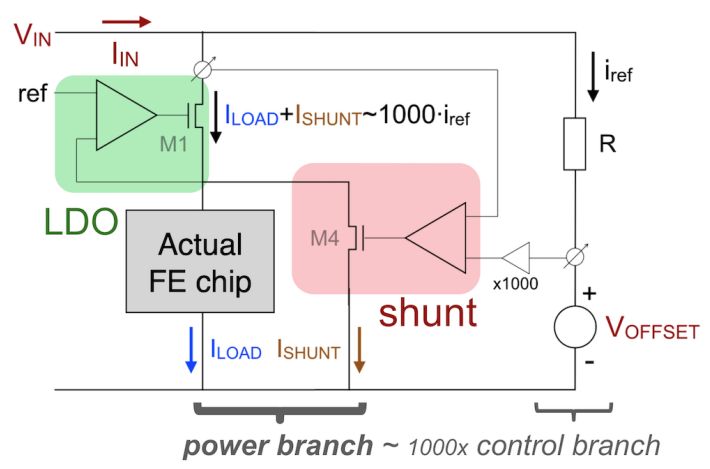

Fig. 6. A sketch illustrating the operating principle of the ShuntLDO device featured in the RD53A prototype.

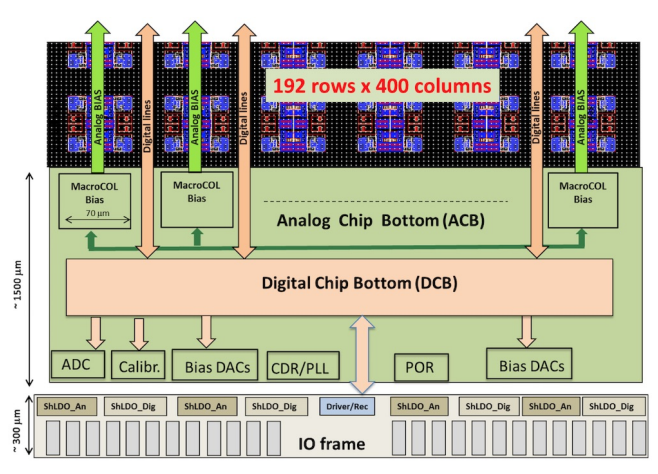

Fig. 7. A simplified floorplan of the RD53A chip. 

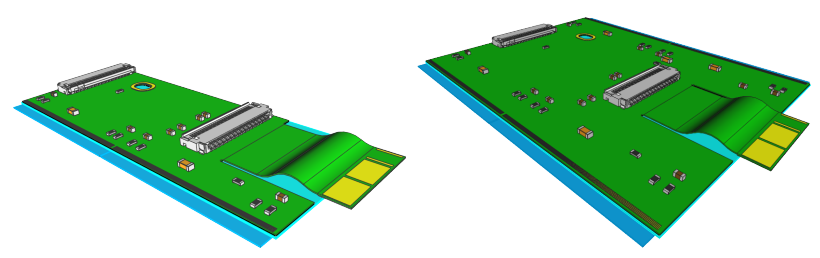

Fig. 8. CAD drawings of $1 \times 2$ (left) e $2 \times 2$ (right) IT pixel modules.

\section{B. The IT Pixel modules}

The IT is based on pixel sensor modules in two flavours, $1 \times 2$ and $2 \times 2$ chips, shown in Fig. 8. Each module is build up by the sensor, bump-bonded to the ROC chips, by a HighDensity Interconnect (HDI) to distribute signals and power and additional minimal elements (e.g. AlN rails) for fixture onto the support structure and to ensure sufficient mechanical strength. No extra ASICs other than the ROC are needed.

The modules are arranged in groups of 8 to 12 into a serial powering chain (sketched in Fig. 9) since this is the only viable approach by which it is possible to feed the $50 \mathrm{~kW}$ needed by the IT while minimizing the passive material.

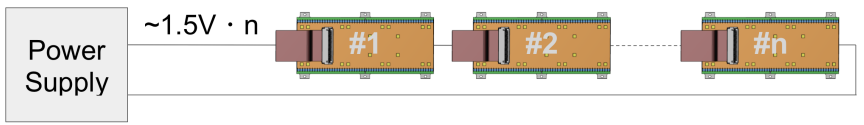

Fig. 9. A sketch illustrating the serial powering concept used within the IT.

\section{IT Luminosity measurement}

Thanks to special extra triggers, TEPX will serve as the main online bunch-by-bunch luminometer for CMS. This functionality will require $\sim 10 \%$ more triggers during normal data taking. As a luminometer TEPX will be operated also before LHC beams are declared stable; in this case all the readout bandwidth is available for the luminosity measurement for which several methods are under study:

- cluster counting that is characterized by a high statistics but suffers from large fake rate;

- multi-hit stub counting using overlaps that would allow for a reduction of the fake rate still maintaining a fairly large statistics;

- track counting that, however, needs processing in the back-end processors.

\section{The OUter Tracker}

As visible in Fig. 1, the Outer Tracker (OT) is organized in six barrel layers and five endcap disks per side that build up the TEDD subsystem. The barrel subsystems are the TBPS and the TB2S, the three innermost and outermost barrel layers, respectively. An innovative 'tilted' geometry is used in the TBPS to optimize the active silicon surface usage with respect to performance.

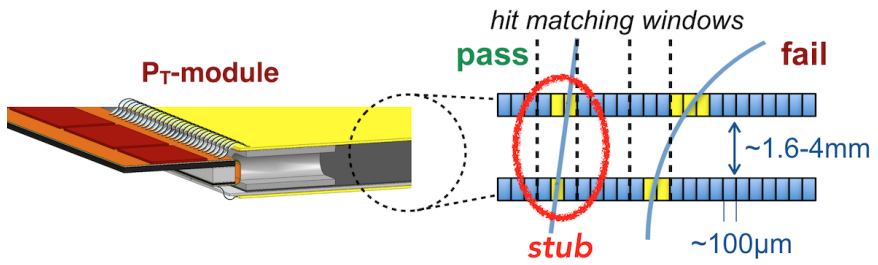

Fig. 10. The concept of the low- $p_{\mathrm{t}}$ hit rejection in closely-spaced sensors where the "selection window" defines an accepted "stub".

\section{A. OT Modules}

The key L1 functionality of the upgraded Tracker, extensively described in [3], [4], [8], is based on data reduction performed on the OT modules, called $p_{\mathrm{t}}$-modules. They are composed of two closely-spaced silicon sensors read out by common front-end ASICs capable of correlating the hits from the two sensors, as sketched in Fig. 10; thanks to the strong magnetic field, hit pairs that form "stubs" compatible with particles above the chosen $p_{\mathrm{t}}$ threshold are selected and dispatched to the L1 trigger system at every bunch crossing. All other hits are stored in the front-end pipelines waiting to be read out when a L1 trigger is received. A uniform $p_{\mathrm{t}}$ threshold throughout the tracking volume is achieved by tuning the acceptance window, programmable in the ASICs, and by choosing different sensor spacings across the Tracker regions. As shown in Fig. 11, the $p_{t}$-module enables a sufficient local data reduction while remaining efficient for the tracks above $2-3 \mathrm{GeV}$ in $p_{\mathrm{t}}$ that are important for the L1 trigger system.

OT modules are standalone units equipped with FE ASICs, Point-of-Load (DC/DC) converter and data link (Low-power GigaBit Transceiver - LpGBT - with laser driver).

Two $p_{\mathrm{t}}$-modules are foreseen, shown in Fig. 12. "2S" modules are composed of two strip sensors each approximately $10 \times 10 \mathrm{~cm}^{2}$ in area, with two rows of $\sim 5 \mathrm{~cm} \mathrm{AC-coupled}$ strips with $90 \mu \mathrm{m}$ pitch. The sensors are mounted such that the strips of the two sensors are parallel to each other. They will be used in the outer regions outside a radius of approximately $60 \mathrm{~cm}$ (in red in Fig. 1). "PS" modules are composed of two sensors each approximately $5 \times 10 \mathrm{~cm}^{2}$ in area, one segmented into approximately $2.5 \mathrm{~cm}$ AC-coupled strips with $100 \mu \mathrm{m}$ pitch, and the other segmented into DC-coupled "macropixels" of size $100 \mu \mathrm{m} \times 1.5 \mathrm{~mm}$.
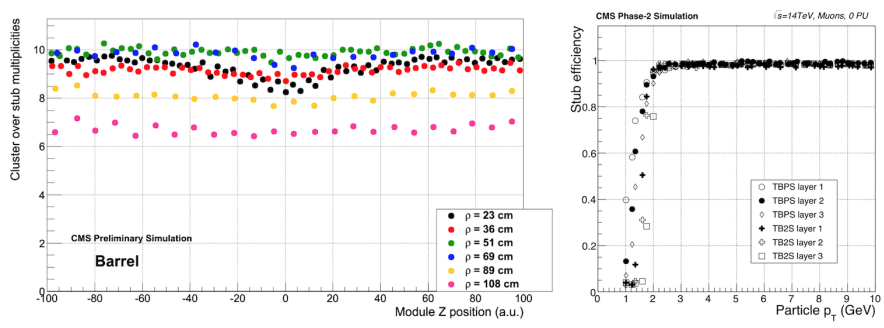

Fig. 11. The $p_{\mathrm{t}}$-module concept performance in the OT barrel: data reduction (left); stub reconstruction efficiency as a function of the particle $p_{\mathrm{t}}$ (right). 

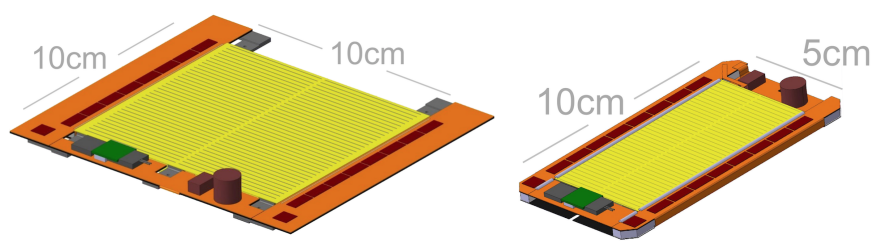

Fig. 12. Three-dimensional view of the assembled $2 \mathrm{~S}$ module (left) and of the PS module (right).

PS modules are deployed in the radial range between $20 \mathrm{~cm}$ and $60 \mathrm{~cm}$, i.e. the inner region (blue in Fig. 1). The macropixels precise measurements of the $z$ coordinate is crucial for primary vertex discrimination at $\mathrm{L} 1$ and for a robust pattern recognition.

Overall the OT consists of 13296 modules for a total of $\sim 192 \mathrm{~m}^{2}$ of $\mathrm{Si}$ sensors with $42 \mathrm{M}$ strips and $170 \mathrm{M}$ macropixels.

\section{B. OT sensors}

Depending on type and final position, OT sensors will be exposed to particle fluences up to $1.5 \cdot 10^{15}\left(1 \mathrm{MeV} n_{\mathrm{eq}}\right) \cdot \mathrm{cm}^{-2}$ and three types of sensors will be used: $5 \mathrm{~cm}$ strips with $90 \mu \mathrm{m}$ for the $2 \mathrm{~S}$ modules, $2.3 \mathrm{~cm}$ with $100 \mu \mathrm{m}$ strips for PS modules and $1467 \mu \mathrm{m}$ macro-pixels with $100 \mu \mathrm{m}$ pitch for the PS modules. These sensors will be n-in-p fabricated out 6" wafers with active thicknesses between 200 and $300 \mu \mathrm{m}$. Final decision on material, thickness and isolation technique is on-going. The nominal bias voltage will be $600 \mathrm{~V}$ with the possibility to increase up to $800 \mathrm{~V}$. As an example, Fig. 13 shows the cluster seed-strip signal as a function of the bias voltage for $2 \mathrm{~S}$ sensor prototypes for HL-LHC fluence figures.

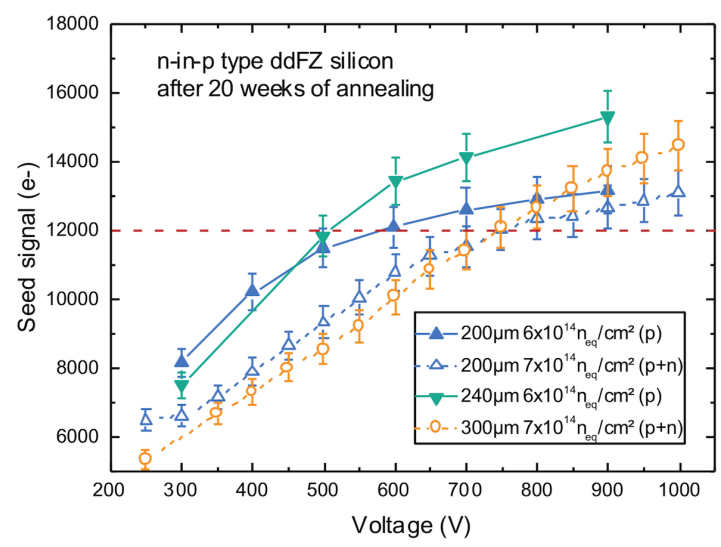

Fig. 13. Cluster seed-strip signal as a function of the bias voltage for $2 \mathrm{~S}$ sensor prototypes for HL-LHC fluence figures.

\section{OT Off-Detector Electronics}

The OT DAQ, controls and power supply chain is sketched in Fig. 14 where the functional elements of the back-end system are also shown. The Data, Trigger \& Control Board

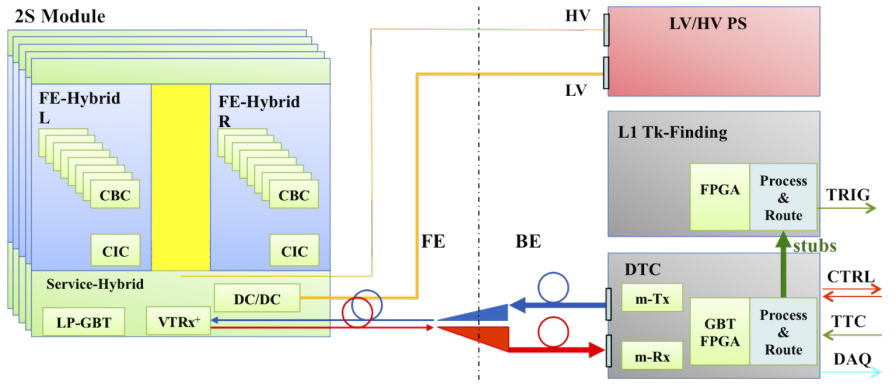

Fig. 14. A sketch the OT DAQ, controls and power supply chain for a OT $2 \mathrm{~S}$ module.

(DTC) sends and receives data from 72 input fibres. This board is a custom development based on off-the-shelf commercial FPGAs and multi-channel optoelectronic transceivers. It processes three detector data streams: Timing and Control, DAQ and Trigger with a global usable bandwidth of 720 (180) Gb/s from (to) detector. The DTC board sends the high- $p_{\mathrm{t}}$ track data selected by the $p_{\mathrm{t}}$-modules, i.e. the stub stream, to the L1 track finding system with $25 \mathrm{~Gb} / \mathrm{s}$ bandwidth [8].

The OT will require $\sim 100 \mathrm{~kW}$ of power that will be supplied to the FE at a voltage of $10-12 \mathrm{~V}$ by a system that will likely be located in the experiment balconies. The power supply will also provide up to $800 \mathrm{~V}$ of bias voltage with up to $2 \mathrm{~mA}$ to the modules. The current design foresees individual supply lines to each detector module that would best match the Tracker modularity and alleviate the need for complex fail-safe redundancy schemes.

\section{OT mechanical structure}

The mechanical concept of the Phase-2 Tracker is the same as for the current Tracker and consists of a support tube at the outermost radius and smaller substructures.

The $2 \mathrm{~S}$ modules that equip the TB2S are mounted onto ladders (see Fig. 15), 12 modules each, then installed in a support wheel that consists of four vertical disks joined by cylinders at the inner and outer radii.

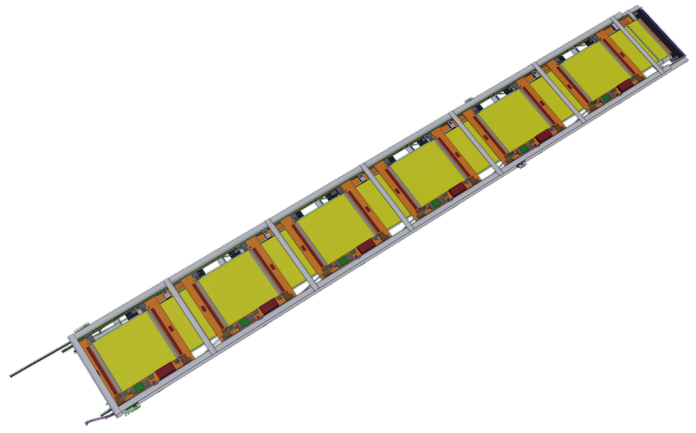

Fig. 15. A TB2S ladder.

The PS modules that equip the central part of the TBPS, shown in Fig. 16, are glued directly onto flat composite structures in which the cooling pipes are embedded. Figure 17 


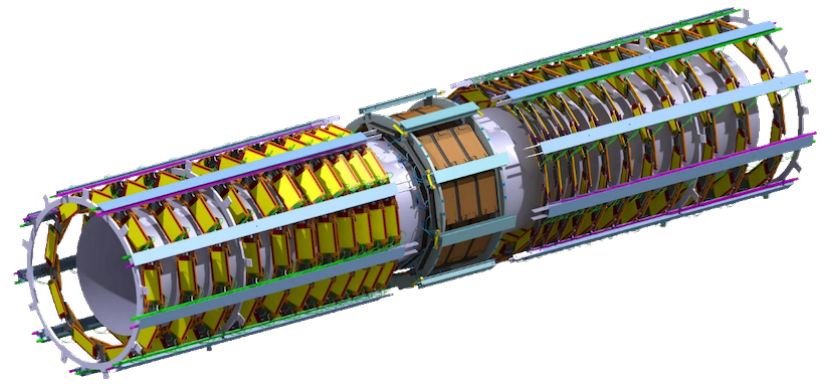

Fig. 16. CAD perspective view of the TBPS.

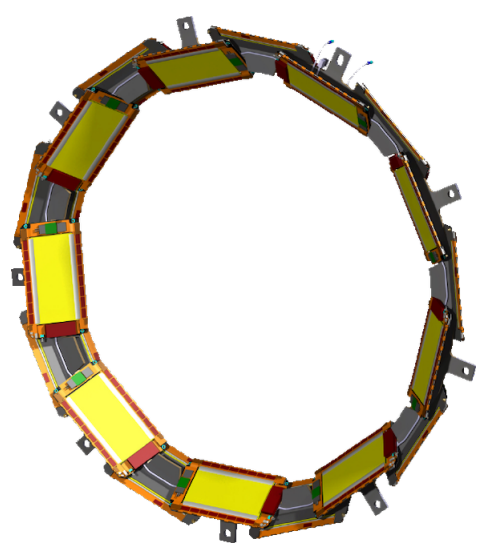

Fig. 17. A TBPS ring with tilted modules.

instead shows the ring structure that enables for the 'tilted' layout concept.

In the endcaps the modules are mounted on flat disks, as shown in Fig. 18. These are split in half-disks, or "dees". Two disks are grouped to form one Double-Disk; ten Double-Disk units will be produced, five for each end-cap or TEDD.

\section{COMMON ASPECTS}

\section{A. Cooling}

The Tracker will be operated and maintained well below $-20^{\circ} \mathrm{C}$ using a two-phase $\mathrm{CO}_{2}$ cooling system running at $-30^{\circ} \mathrm{C}$. The total power dissipated by the Tracker within the tracking volume is estimated to be around $\sim 100 \mathrm{~kW}$ for the OT and $\sim 50 \mathrm{~kW}$ for the IT. As sketched in Fig. 19 the cooling system relies on five identical $50 \mathrm{~kW}$ cooling plants. For redundancy, one is kept always in stand-by ready to take over if needed.

\section{B. Data Flow}

The readout and control system is schematically represented in Fig. 20. The OT, via the DTC, will feed L1 Track Trigger processors with track trigger data at $40 \mathrm{MHz}$. OT and IT will be fully readout on the receipt of a L1 accept signal at a maximum rate of $750 \mathrm{kHz}$. As above explained, in parallel, special L1 'lumi' triggers will be issued to IT TEPX for instantaneous luminosity measurements. It is estimated that

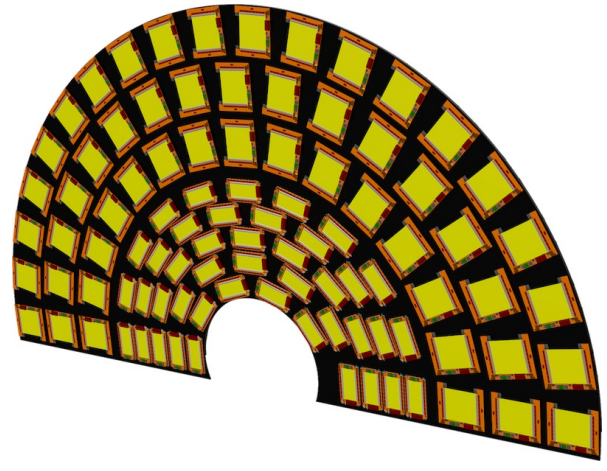

Fig. 18. A TEDD dee.

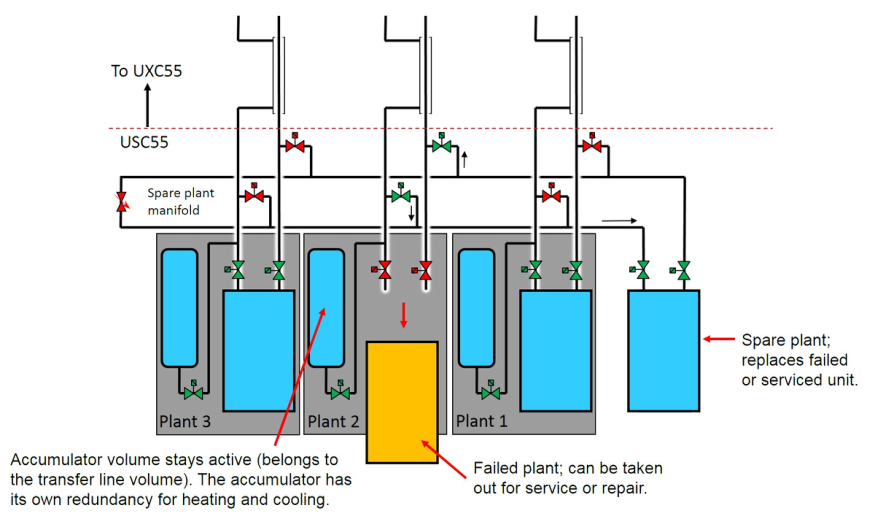

Fig. 19. A sketch of the cooling system illustrating the redundant configuration of the cooling plants deployment.

these triggers should have a $75 \mathrm{kHz}$ rate to ensure sufficient statistics for the measurements.

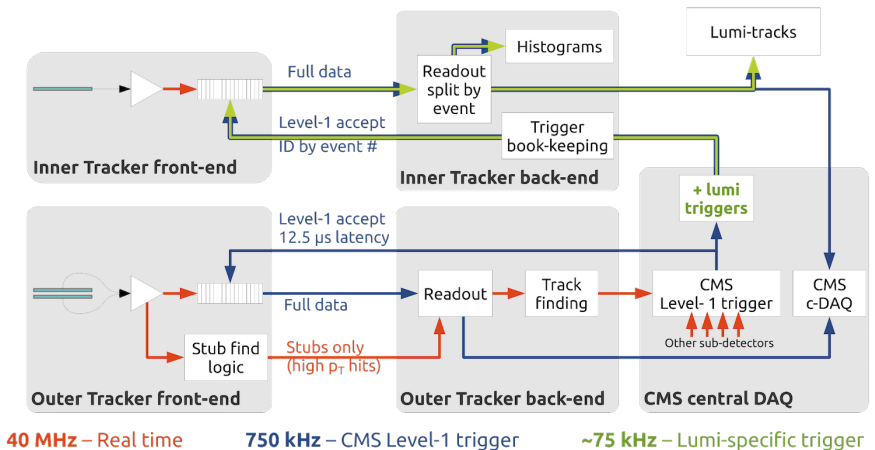

Fig. 20. A schematic representation of the IT and OT data and trigger flow.

\section{Material Budget}

The upgrade project aims at a significant reduction of the impact of the tracker material with respect to the current Phase-1 Tracker. In fact, overall, the estimated material budget of the upgraded Tracker shows a significant reduction, in particular around $|\eta|=1.5$, as visible in Fig. 21 where the Phase-2 vs. Phase-1 comparison is shown. 

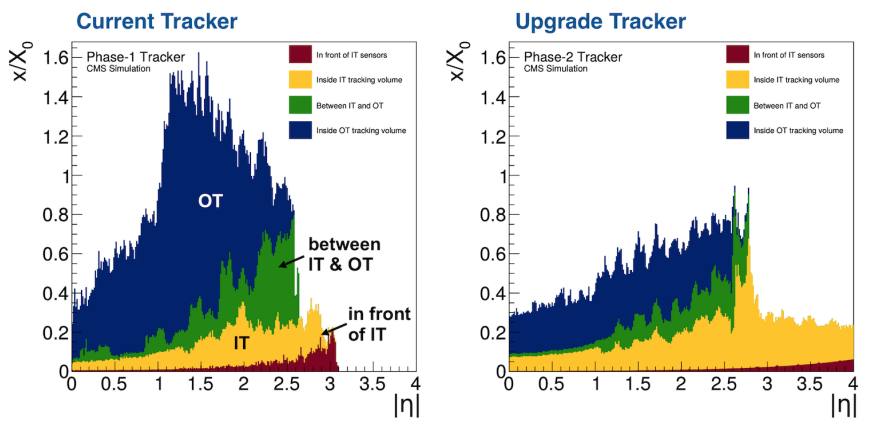

Fig. 21. Material budget comparison for the Phase-1 and Phase-2 tracker detectors.

\section{Performance}

Some highlights of the expected offline tracking performance [4] are discussed. The track reconstruction efficiency for the Phase-2 detector at a PU of 140 and 200 for simulated $t \bar{t}$ events, shown in Fig. 22 for $t \bar{t}$ together with the expected fake rate, is as good as the Phase-1 efficiency at a PU of 50 events and extends the $|\eta|$ range by about 1.5 units thanks to the foreseen extension of the pixel detector. The fraction of reconstructed ghost tracks is higher but still adequate for the CMS event reconstruction.

The impact of the much better granularity and the reduced passive material on physics performance is well visible in Fig. 23 where the resolution on $p_{\mathrm{t}}$ and the transverse impact parameter are reported as a function of the pseudorapidity in comparison between the Phase-1 and upgraded Phase- 2 tracker detectors.

The ability to perform track reconstruction in dense environment as the jet core is an important figure of merit for a tracking device at a pp collider. The resolutions of jet-related observables very much depend on the possibility to disentangle, e.g. by using vertex information, the tracks belonging to the jet with respect to PU tracks accidentally close-by. The clear improvement in this respect is visible in Fig. 24 where the track reconstruction efficiency as a function of the distance $\Delta R=\sqrt{(\Delta \eta)^{2}+(\Delta \phi)^{2}}$ from the jet axis is shown for the Phase-1 and Phase-2 tracker detectors.
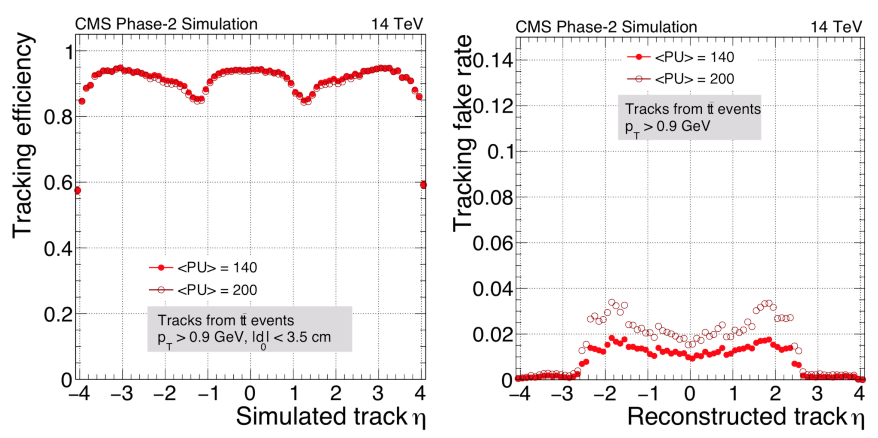

Fig. 22. Tracking performance as a function of $|\eta|$ on simulated $t \bar{t}$ events at PU 140 and 200: efficiency (left) and fake rate (right).
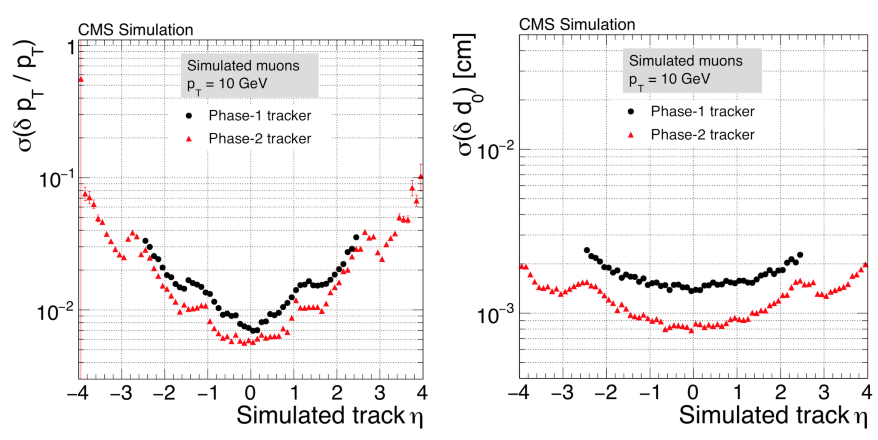

Fig. 23. Comparison of reconstructed track parameter resolutions between the Phase-1 tracker and the upgraded, Phase-2, tracker: transverse momentum (left) and transverse impact parameter (right).

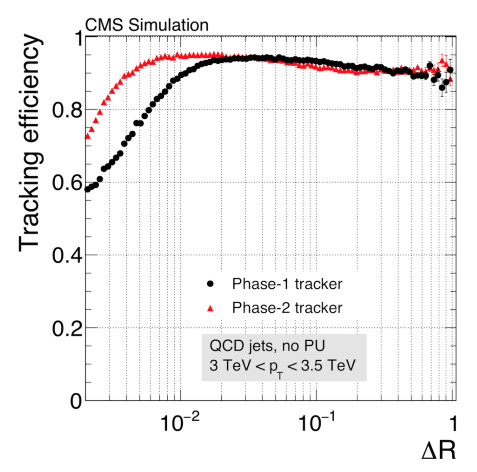

Fig. 24. Track reconstruction efficiency as a function of the distance $\Delta R=$ $\sqrt{(\Delta \eta)^{2}+(\Delta \phi)^{2}}$ from the jet axis for the Phase-1 and upgraded Phase-2 tracker detectors.

\section{CONCLUSIONS}

The Phase-2 Tracker upgrade for HL-LHC consists of a replacement of the whole Tracker detector. The development of the new Tracker is well advanced. The detector layout is being optimized and finalized and several R\&D activities are ongoing on sensors, electronics and mechanics. The project will soon enter into parts production and integration.

\section{REFERENCES}

[1] The CMS Collaboration, The CMS experiment at the CERN LHC, JINST 3, S08004 (2008).

[2] The CMS Collaboration, CMS Technical Design Report for the Pixel Detector Upgrade, CERN-LHCC-2012-016.

[3] The CMS Collaboration, Technical Proposal for the Phase-II Upgrade of the CMS Detector, CERN-LHCC-2015-010; LHCC-P-008.

[4] The CMS Collaboration, The Phase-2 Upgrade of the CMS Tracker Technical Design Report, CERN-LHCC-2017-009, CMS-TDR-014.

[5] G. Steinbrueck on behalf of the CMS tracker group Development of CMS pixel sensors for extreme fluences at $H L-L H C$, this conference.

[6] For more informations about RD53: http://rd53.web.cern.ch/rd53/

[7] S. Orfanelli, D. Koukola on behalf of the CMS tracker group Serial Powering for Inner Tracker Detectors at the HL-LHC, this conference.

[8] F. Palla on behalf of the CMS tracker group Level-1 track finding with an all-FPGA system at CMS for the HL-LHC, this conference. 